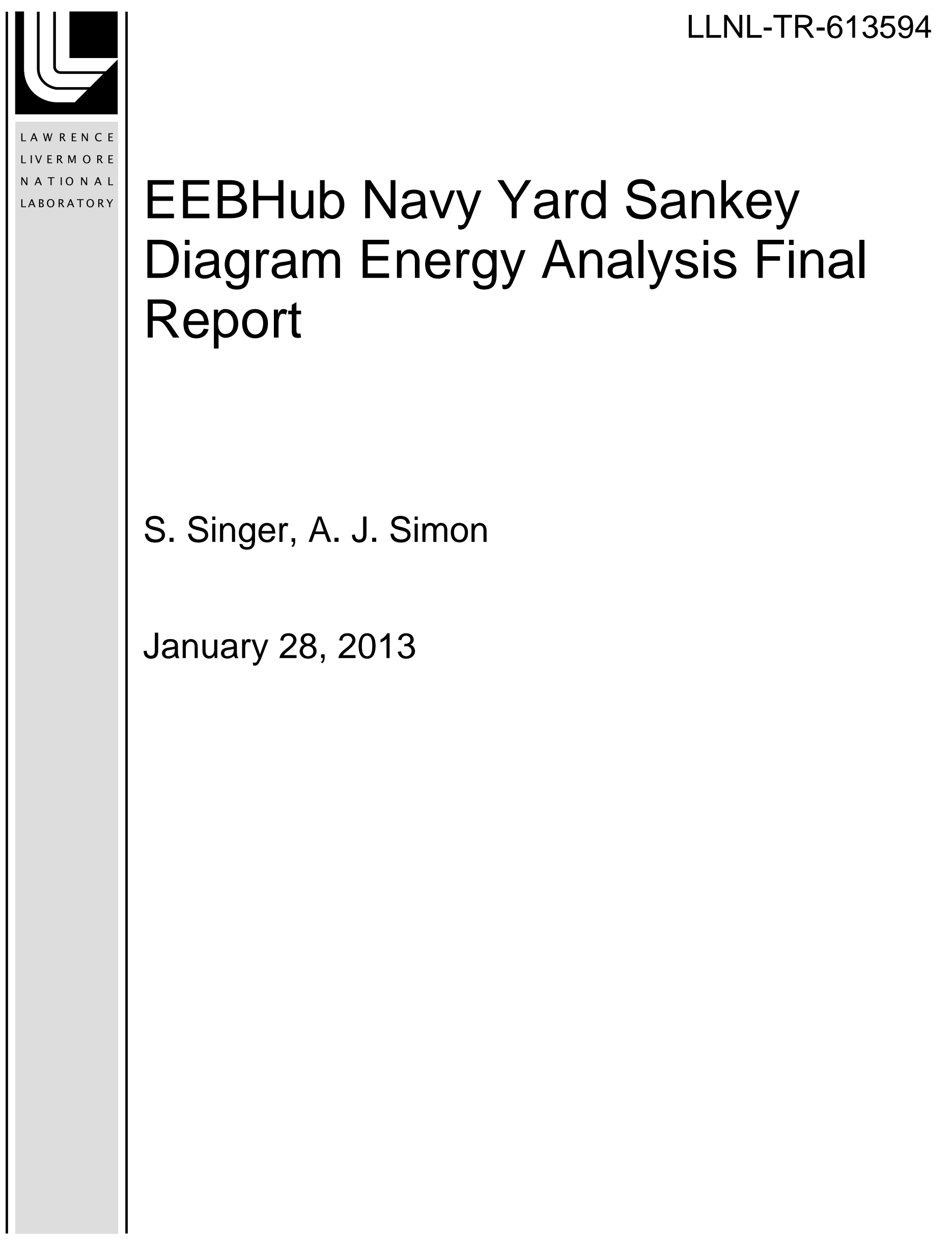


This document was prepared as an account of work sponsored by an agency of the United States government. Neither the United States government nor Lawrence Livermore National Security, LLC, nor any of their employees makes any warranty, expressed or implied, or assumes any legal liability or responsibility for the accuracy, completeness, or usefulness of any information, apparatus, product, or process disclosed, or represents that its use would not infringe privately owned rights. Reference herein to any specific commercial product, process, or service by trade name, trademark, manufacturer, or otherwise does not necessarily constitute or imply its endorsement, recommendation, or favoring by the United States government or Lawrence Livermore National Security, LLC. The views and opinions of authors expressed herein do not necessarily state or reflect those of the United States government or Lawrence Livermore National Security, LLC, and shall not be used for advertising or product endorsement purposes.

This work performed under the auspices of the U.S. Department of Energy by Lawrence Livermore National Laboratory under Contract DE-AC52-07NA27344. 


\section{EEBHUB NAVY YARD SANKEY DIAGRAM ENERGY ANALYSIS FINAL REPORT \\ LLNL-TR-613594}

Suzanne L. Singer, A.J. Simon

Lawrence Livermore National Laboratory

January 28, 2013 


\section{Contents}

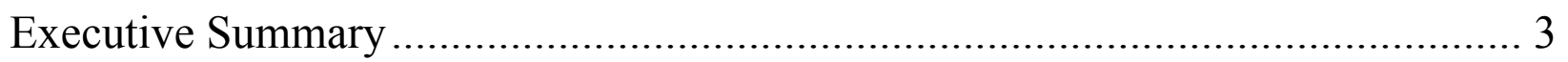

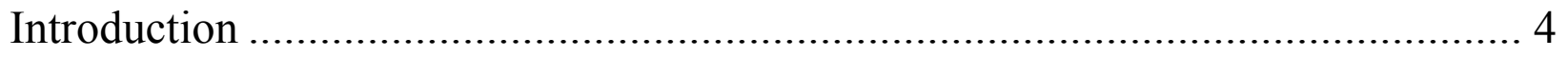

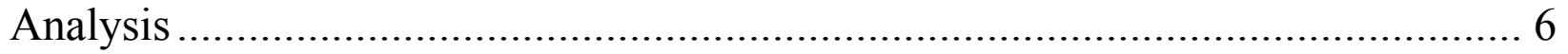

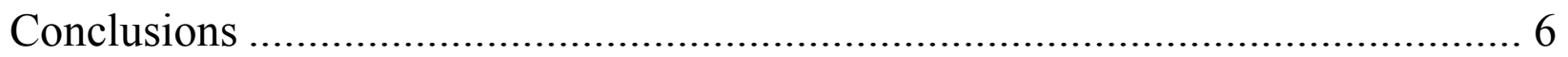

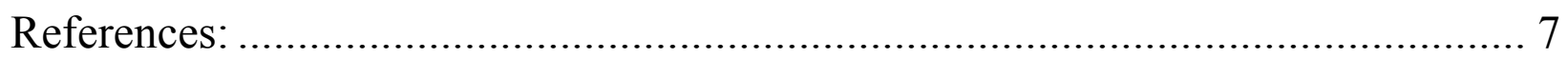

Appendix A: Energy Component Data for Navy Yard Sankey Diagrams............... 8

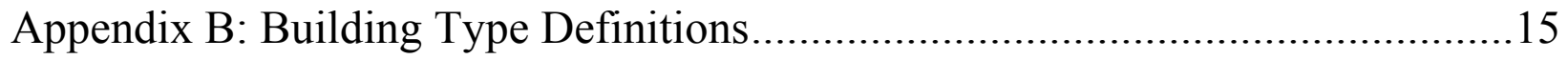




\section{Executive Summary}

The Energy Efficient Buildings Hub (EEBHub) uses Sankey diagrams to visually analyze energy uses among buildings within the Navy Yard. This report describes the preliminary steps taken to incorporate multiple buildings in a district energy analysis, which can eventually be expanded to the Greater Philadelphia Region.

The Navy Yard analysis incorporates $\sim 170$ buildings, with energy flows and data broken down to building type and end use based on Energy Information Administration (EIA) commercial data for buildings. The electricity and natural gas consumption is provided by EEBHub. The largest end uses of fuel consumption are space heating ( 140 MBtu) and lighting ( 72 MBtu). The natural gas consumption is heavily used in the service areas ( 120 MBtu) mainly for space heating. The electricity consumption is more evenly spread out among building type with the largest uses in office space and other (description in Appendix B).

As the Navy Yard data gets updated, there are opportunities to analyze the Navy Yard in other formats. The results of this preliminary analysis are to identify large sources of energy consumption, recognize gaps in data, and use Sankey visualizations to address the goals of the EEBHub. 


\section{Introduction}

The Energy Efficient Buildings Hub (EEBHub) requested visualization of energy flows in Sankey format for buildings in the Navy Yard. These diagrams provide a compact representation of quantitative information from multiple sources of data. This work will lay the groundwork for visualizing building energy use within the Greater Philadelphia Region, which is instrumental to demonstrating the success of EEBHub. A quantitative understanding of interrelated energy flows helps to identify opportunities to deploy energy saving technologies.

The Sankey diagrams address several objectives identified by EEBHub

- Integrate design, construction, commissioning, and operation of buildings

- Integrate energy saving technologies for whole building system solutions at the Navy Yard and elsewhere in the region

- Inform, train, and educate people (i.e. policy makers, community, workforce) about proven energy saving strategies and technologies

This report describes a preliminary analysis of energy usage and flows among buildings in the Navy Yard. The starting point of this project was to sketch a qualitative Sankey diagram that identifies important sources of energy consumption. We chose to analyze data by building primary use type to estimate end use consumption of the buildings. Details of the analysis are described in Appendix A.

The two sources of data used in this analysis are:

- Energy Information Administration (EIA) tables that list major components of energy consumption for commercial buildings as displayed in Tables A2 and A3.

- Navy Yard data that includes building use type of 528 entities, annual electricity usage for 89 buildings, and annual natural gas usage for 48 buildings.

As the understanding of the Navy Yard energy use is updated and refined, Sankey diagrams can be updated and modified. 


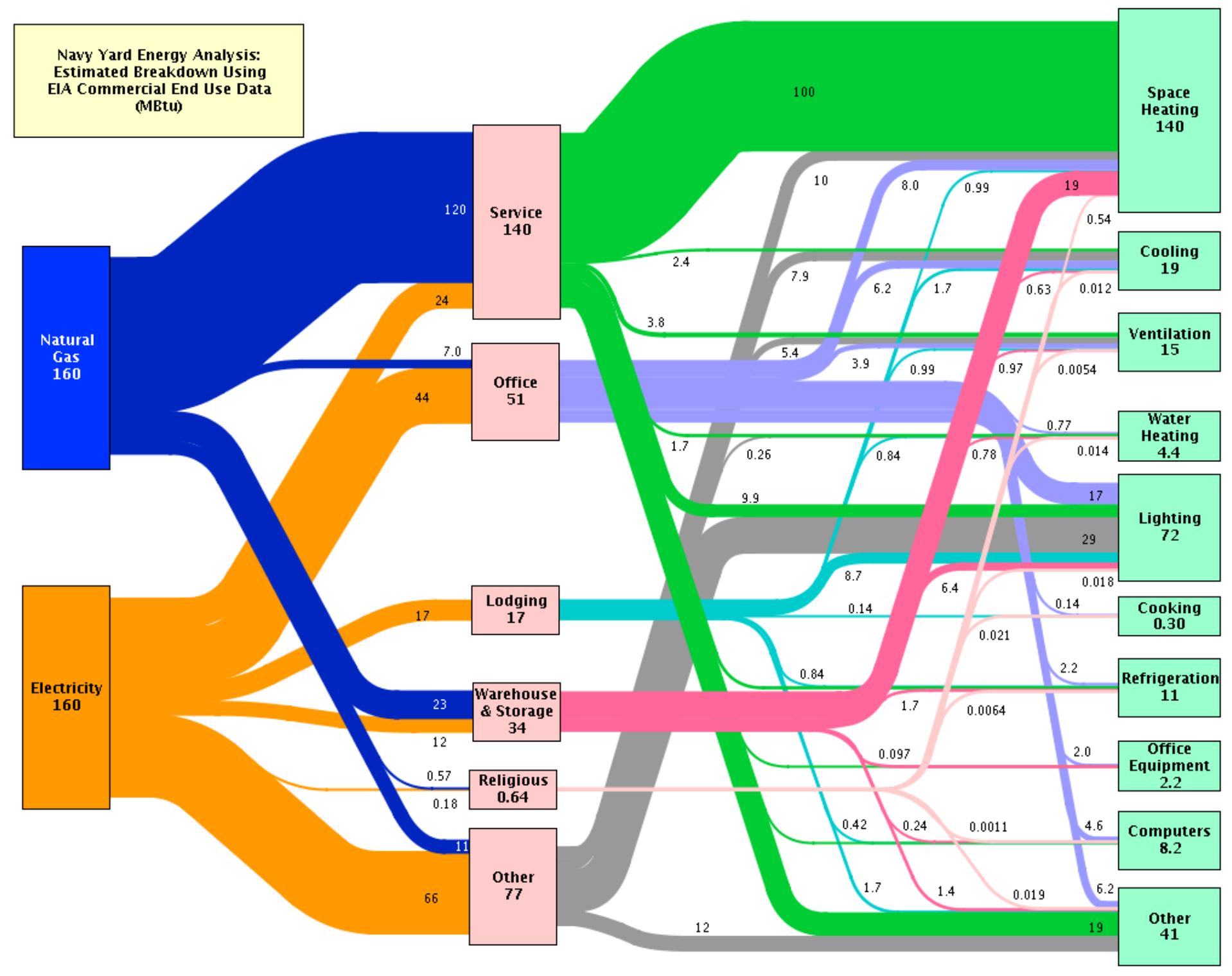

Figure 1 Navy Yard Energy Analysis: Estimated using EIA Commercial End Use Data (MBtu). 


\section{Analysis}

The starting point for this analysis was the 1/2010 - 12/2011 energy consumption data for all Navy Yard buildings collected by the electric and gas utilities that serve the Navy Yard. These data were organized into a single spreadsheet containing electricity and natural gas use for $\sim 170$ existing buildings. Of these buildings, $\sim 100$ consume metered electricity, and $\sim 60$ consume natural gas. Such a large number of buildings cannot be individually depicted (each with its own "box" for energy use) on a single Sankey Diagram. Therefore, we chose to aggregate the buildings in the Navy Yard by usage type (lodging, office, warehouse, etc.)

Because most of the buildings at the Navy Yard are not sub-metered, we needed a way to estimate the fractions of electricity and gas that are put to various end-uses (heating, lighting, etc.) in each building. We chose to use EIA's 2003 Commercial Buildings Energy Consumption Survey data to estimate the end-use energy loads in each building.

For each building at the Navy Yard, we multiplied the quantity of natural gas consumed (as metered) by the EIA-estimated fraction of natural gas energy applied to each end use in that building type. Similarly, we multiplied the quantity of electricity consumed (as metered) by the EIA-estimated fraction of electricity applied to each end use in the building's type. See Appendix A for the fractions of consumption applied.

We then combined these flows ( 2 energy types used in 10 end-uses each) into a maximum of 10 flows from each building type to the end uses. In this way, the total energy consumed for each end use can be compared with the total energy consumed for each type of building.

It should be noted, however, that this visualization is a draft, and based on preliminary energy consumption data provided by the utilities that serve the Navy Yard. While all data have been normalized to reflect annual energy use, the data are from the most recent time period available, and are not based on a statistically representative sample of historical values.

\section{Conclusions}

The Navy Yard Sankey diagrams depict estimated energy flows based on end uses of commercial buildings. The estimated end use values are preliminary, so they are not specific to Philadelphia or the Navy Yard.

The Sankey diagram does, however, provide a framework in which to discuss future submetering efforts at EEBHub. When a majority of the buildings are instrumented, this diagram will transition from an estimate of energy consumption to a compact form of energy use reporting.

Specific actions may be taken to speed this transition. For example, the Hub may want to focus on a specific building class (e.g. office buildings), and could choose to focus its efforts on the specific subset of buildings belonging to that class. Alternatively, the Hub might choose to focus on the largest end uses of energy: heating and lighting. 
If available, the Hub might also choose to analyze historical data and compare year-over-year energy use, or depict the evolution of the Navy Yard's energy distribution systems (transition from steam to gas heating, for example.)

Ultimately, this preliminary graphic serves as a strawman template for a regional energy dashboard, whose key attributes will be defined by EEBHub personnel and other stakeholders.

\section{References:}

Energy Information Administration. 2003 Commercial Buildings Energy Consumption Survey: Energy End-Use Consumption Tables. Table E3 and E7. 


\section{Appendix A: Energy Component Data for Navy Yard Sankey Diagrams}

A breakdown of energy data by commercial building type and end use is published by the Energy Information Administration (EIA) ${ }^{1}$. The data is separated into seven categories of principal building activity (i.e. food sales, lodging, office, religious worship, service, warehouse and storage, and other). The EIA data also breaks down major fuel consumption to end use (Table A1).

Data for the Navy Yard buildings includes the primary building type and estimated electricity and natural gas consumption ${ }^{2}$, and is currently being updated by collaborators in the EEBHub. Older versions of the data may have significant uncertainties associated with the data. Possible reasons include: buildings have changed from steam to natural gas for heating, and permission for data collection is still needed for some buildings.

The steps taken to evaluate the building data:

1) From the EIA tables, calculate the fraction of end use type for each building type (i.e. lighting end use takes $\sim 53 \%$ of the total electricity consumption for lodging building type). Table A1 lists building and end use type used in the Navy Yard analysis. Table A2 and Table A3 show the fraction of consumption by each end use type.

2) The Navy Yard data is separated into building type, shown in Table A4. The electricity and natural gas data are broken down into end use by making them proportional to EIA end use data calculated in step 1. These estimates of fuel use are shown in Table A5.

Table A1 Building type and End Use categories used for the Sankey Diagram

\begin{tabular}{|l|l|l|}
\hline Building Type & $\begin{array}{l}\text { Electricity Consumption } \\
\text { End Use }\end{array}$ & $\begin{array}{l}\text { Natural Gas Consumption } \\
\text { End Use }\end{array}$ \\
\hline food sales & Space heating & Space heating \\
lodging & Water heating & Water heating \\
office & Cooking & Ooking \\
religious worship & Other \\
service & Cooling & \\
warehouse \& storage & Ventilation & \\
other (incl. non-categorized) & Lighting & \\
& Refrigeration & \\
& Office Equipment & \\
& Computers & \\
\end{tabular}

\footnotetext{
${ }^{1}$ source: EIA Major Fuel Consumption (Btu) by End Use for Non-Mall Buildings, 2003

2 [Navy Yard data was taken from the file provided in email from Robert Leicht to A.J. Simon

"NavyYardData_UTRC_29Mar.xIsx", 9/19/12]
} 
Table A2 End Use Fraction of Total Natural Gas Consumption for Building Type, Modified from EIA Table E7: Natural Gas Consumption (Btu) by End Use for Non-Mall Buildings, 2003.

\begin{tabular}{|l|c|c|c|c|}
\cline { 2 - 5 } \multicolumn{1}{c|}{} & Space heating & Water heating & Cooking & Other \\
\hline Food Sales & 0.69 & 0.05 & 0.21 & $\mathrm{Q}^{3}$ \\
\hline Lodging & 0.30 & 0.58 & 0.07 & $\mathrm{Q}$ \\
\hline Retail & 0.92 & 0.03 & 0.03 & 0.02 \\
\hline Office & 0.86 & 0.05 & 0.01 & 0.09 \\
\hline Religious Worship & 0.94 & 0.02 & 0.04 & $\mathrm{Q}$ \\
\hline Service & 0.86 & 0.01 & $\mathrm{Q}$ & 0.12 \\
\hline Warehouse \& Storage & 0.84 & 0.03 & $\mathrm{Q}$ & $\mathrm{Q}$ \\
\hline Other & 0.83 & 0.02 & $\mathrm{Q}$ & 0.14 \\
\hline Vacant & 0.93 & $\mathrm{Q}$ & $\mathrm{Q}$ & $\mathrm{Q}$ \\
\hline
\end{tabular}

Table A3 End Use Fraction of Total Electricity Consumption for Building Type, modified from EIA Table E3: Electricity

\begin{tabular}{|l|c|c|c|c|c|c|c|c|c|c|}
\multicolumn{1}{l|}{} & $\begin{array}{c}\text { Space } \\
\text { heating }\end{array}$ & Cooling & Ventilation & $\begin{array}{c}\text { Water } \\
\text { heating }\end{array}$ & Lighting & Cooking & Refrigeration & $\begin{array}{c}\text { Office } \\
\text { Equipment }\end{array}$ & Computers & Other \\
\hline Food Sales & 0.03 & 0.06 & 0.03 & $\mathrm{Q}$ & 0.22 & 0.01 & 0.57 & 0.01 & 0.01 & 0.05 \\
\hline Lodging & 0.06 & 0.10 & 0.06 & 0.05 & 0.53 & 0.01 & 0.05 & $\mathrm{Q}$ & 0.03 & 0.10 \\
\hline Retail & 0.03 & 0.12 & 0.08 & 0.01 & 0.53 & $\left(^{*}\right)^{4}$ & 0.10 & 0.01 & 0.02 & 0.10 \\
\hline Office & 0.05 & 0.14 & 0.09 & 0.01 & 0.39 & 0.001 & 0.05 & 0.04 & 0.10 & 0.13 \\
\hline $\begin{array}{l}\text { Religious } \\
\text { Worship }\end{array}$ & 0.05 & 0.18 & 0.08 & $\left({ }^{*}\right)$ & 0.27 & $\left(^{*}\right)$ & 0.10 & $\left(^{*}\right)$ & 0.02 & 0.29 \\
\hline Service & 0.04 & 0.10 & 0.16 & $\left(^{*}\right)$ & 0.42 & $\mathrm{Q}$ & 0.06 & 0.01 & 0.02 & 0.19 \\
\hline $\begin{array}{l}\text { Warehouse } \\
\text { \& Storage }\end{array}$ & 0.02 & 0.05 & 0.08 & 0.01 & 0.54 & $\mathrm{Q}$ & 0.15 & 0.01 & 0.02 & 0.12 \\
\hline Other & 0.02 & 0.12 & 0.08 & $\mathrm{Q}$ & 0.44 & $\mathrm{Q}$ & 0.08 & $\mathrm{Q}$ & 0.04 & 0.17 \\
\hline Vacant & 0.07 & 0.13 & 0.07 & $\mathrm{Q}$ & 0.27 & $\mathrm{Q}$ & $\left(^{*}\right)$ & $\mathrm{Q}$ & $\left({ }^{*}\right)$ & 0.47 \\
\hline
\end{tabular}

\footnotetext{
${ }^{3} \mathrm{Q}=$ Data withheld because fewer than 20 buildings were sampled for any cell, or because the Relative Standard Error (RSE) was greater than 50 percent for a cell in the "Total" column.

${ }^{4}\left({ }^{*}\right)=$ Value rounds to zero in the units displayed.
} 
Table A4 Navy Yard Building Number, Primary Use, Electricity, and Natural Gas Annual Use

\begin{tabular}{|c|c|c|c|c|}
\hline Index & Description & Primary Building Use & $\begin{array}{c}\text { Annual Electric } \\
\text { Use (kWh) }\end{array}$ & $\begin{array}{c}\text { Annual NG Use } \\
\text { (CCF) } \\
\end{array}$ \\
\hline 1 & B1 & Office & 0 & 0 \\
\hline 3 & 3 & Office & 171,837 & 25,203 \\
\hline 4 & B4 & Office & $2,344,250$ & 58 \\
\hline 6 & B6 & Office & 388,838 & 18,150 \\
\hline 7 & B7 & Office & $3,871,650$ & 0 \\
\hline 9 & 9 & Service & 0 & 0 \\
\hline 10 & B10 & Nonrefrigerated warehouse & 598,939 & 0 \\
\hline 11 & B11 & Office & $1,461,081$ & 0 \\
\hline 12 & B12 & Service & 0 & 0 \\
\hline 14 & 14 & Service & 15,271 & 0 \\
\hline 15 & B15 & Office & 0 & 0 \\
\hline 16 & B16 & Service & $1,227,113$ & 0 \\
\hline 17 & 17 & Service & 83,550 & 0 \\
\hline 18 & 18 & Service & 0 & 0 \\
\hline 19 & 19 & Service & 0 & 0 \\
\hline 20 & $\mathrm{~B} 20$ & Service & 0 & 30,021 \\
\hline 21 & 21 & Other & 0 & 0 \\
\hline 22 & B22 & Service & 0 & 0 \\
\hline 23 & $\mathrm{~B} 23$ & Service & 22,380 & $1,050,786$ \\
\hline 25 & B25 & Service & 0 & 0 \\
\hline 26 & B26 & Nonrefrigerated warehouse & 968,941 & 5,947 \\
\hline 29 & 29 & Other & 0 & 899 \\
\hline 40 & 40 & Other & 0 & 0 \\
\hline 41 & B41 & Service & 38,693 & 0 \\
\hline 44 & B44 & Service & 0 & 1,868 \\
\hline 47 & 47 & Other & 0 & 0 \\
\hline 49 & B49 & Office & 0 & 0 \\
\hline 56 & B56 & Service & 224,605 & 4,254 \\
\hline 57 & B57 & Service & 360,555 & 0 \\
\hline 68 & B68 & Service & 45,183 & 0 \\
\hline 69 & B69 & Service & 35,542 & 1,868 \\
\hline 77 & 77 & Other & $6,458,232$ & 105,537 \\
\hline 80 & 80 & Other & 0 & 0 \\
\hline 82 & 82 & Other & 0 & 0 \\
\hline 83 & 83 & Office & 0 & 0 \\
\hline 84 & 84 & Nonrefrigerated warehouse & 252,800 & 0 \\
\hline 87 & B87 & Service & 0 & 39,599 \\
\hline 93 & 93 & Other & 577,625 & 0 \\
\hline 94 & B94 & Service & 0 & 0 \\
\hline
\end{tabular}




\begin{tabular}{|c|c|c|c|c|}
\hline 95 & B95 & Nonrefrigerated warehouse & 0 & 0 \\
\hline 96 & B96 & Service & 0 & 0 \\
\hline 100 & B100 & Office & 408,570 & 8,522 \\
\hline 101 & B101 & Office & 877,824 & 16,525 \\
\hline 104 & 104 & Other & 0 & 0 \\
\hline 120 & 120 & Nonrefrigerated warehouse & 180,000 & 0 \\
\hline 121 & B121 & Office & 85,543 & 0 \\
\hline 122 & B122 & Nonrefrigerated warehouse & 0 & 0 \\
\hline 139 & B139 & Service & 0 & 0 \\
\hline 455 & 455 & Other & 0 & 0 \\
\hline 480 & B480 & Office & 53,077 & 0 \\
\hline 483 & 483 & Other & 0 & 0 \\
\hline 487 & 487 & Other & 14,585 & 0 \\
\hline 489 & 489 & Other & 0 & 0 \\
\hline 500 & 500 & Office & 0 & 0 \\
\hline 501 & B501 & Office & 0 & 0 \\
\hline 519 & 519 & Other & 0 & 0 \\
\hline 520 & 520 & Service & 0 & 0 \\
\hline 526 & B526 & Service & 0 & 0 \\
\hline 529 & 529 & Service & 19,609 & 0 \\
\hline 530 & 530 & Service & 0 & 0 \\
\hline 531 & 531 & Service & 0 & 0 \\
\hline 542 & B542 & Nonrefrigerated warehouse & 638,100 & 33,761 \\
\hline 543 & B543 & Nonrefrigerated warehouse & 0 & 0 \\
\hline 545 & B545 & Service & 78,824 & 0 \\
\hline 546 & B546 & Service & 0 & 0 \\
\hline 549 & 549 & Other & 829,000 & 0 \\
\hline 567 & B567 & Nonrefrigerated warehouse & 46,166 & 0 \\
\hline 574 & 574 & Other & 50,801 & 0 \\
\hline 590 & 590 & Other & 0 & 0 \\
\hline 592 & B592 & Service & 626,400 & 0 \\
\hline 602 & 602 & Other & $8,638,424$ & 0 \\
\hline 603 & 603 & Nonrefrigerated warehouse & 0 & 0 \\
\hline 605 & $\mathrm{~B} 605$ & Nonrefrigerated warehouse & 25,420 & 0 \\
\hline 608 & 608 & Office & 0 & 0 \\
\hline 611 & B611 & Nonrefrigerated warehouse & 16,096 & 0 \\
\hline 613 & $\mathrm{~B} 613$ & Refrigerated warehouse & 45,600 & 0 \\
\hline 615 & $\mathrm{~B} 615$ & Office & 56,343 & 0 \\
\hline 616 & 616 & Other & 0 & 0 \\
\hline 618 & 618 & Other & 0 & 0 \\
\hline 620 & B620 & Nonrefrigerated warehouse & 0 & 136,386 \\
\hline
\end{tabular}




\begin{tabular}{|c|c|c|c|c|}
\hline 621 & 621 & Other & 0 & 0 \\
\hline 622 & 622 & Other & 0 & 0 \\
\hline 623 & B623 & Service & 44,648 & 3,877 \\
\hline 624 & 624 & Office & 0 & 0 \\
\hline 626 & 626 & Other & 0 & 0 \\
\hline 633 & 633 & Other & $1,964,131$ & 2,194 \\
\hline 634 & $\mathrm{~B} 634$ & Nonrefrigerated warehouse & 0 & 0 \\
\hline 636 & 636 & Office & 0 & 0 \\
\hline 640 & 640 & Office & 0 & 0 \\
\hline 643 & B643 & Service & 6,832 & 0 \\
\hline 649 & B649 & Religious worship & 19,485 & 5,626 \\
\hline 653 & B653 & Nonrefrigerated warehouse & 7,701 & 0 \\
\hline 661 & 661 & Other & 0 & 0 \\
\hline 662 & 662 & Other & 0 & 0 \\
\hline 664 & 664 & Other & 27,548 & 0 \\
\hline 666 & 666 & Other & 0 & 0 \\
\hline 668 & 668 & Service & 0 & 0 \\
\hline 669 & B669 & Service & 0 & 0 \\
\hline 670 & 670 & Other & 0 & 0 \\
\hline 677 & 677 & Other & 4,800 & 0 \\
\hline 679 & B679 & Service & 35,560 & 0 \\
\hline 685 & 685 & Other & 0 & 0 \\
\hline 694 & 694 & Nonrefrigerated warehouse & 166,282 & 17,357 \\
\hline 712 & B712 & Service & 182,310 & 0 \\
\hline 731 & 731 & Other & 0 & 0 \\
\hline 733 & B733 & Service & 0 & 0 \\
\hline 738 & B738 & Service & 0 & 0 \\
\hline 739 & 739 & Other & 135,402 & 0 \\
\hline 744 & 744 & Other & 0 & 0 \\
\hline 745 & 745 & Other & 0 & 0 \\
\hline 753 & 753 & Nonrefrigerated warehouse & 242,700 & 27,894 \\
\hline 754 & B754 & Service & 0 & 0 \\
\hline 756 & 756 & Service & 0 & 0 \\
\hline 763 & B763 & Nonrefrigerated warehouse & 264,838 & 0 \\
\hline 766 & 766 & Other & 0 & 0 \\
\hline 770 & 770 & Other & 0 & 0 \\
\hline 771 & 771 & Other & 0 & 0 \\
\hline 772 & B772 & Service & 87,250 & 5,993 \\
\hline 776 & 776 & & 0 & 0 \\
\hline 778 & 778 & Service & 219,260 & 0 \\
\hline 793 & 793 & Other & 0 & 0 \\
\hline
\end{tabular}




\begin{tabular}{|c|c|c|c|c|}
\hline 796 & 796 & Other & 55,196 & 0 \\
\hline 811 & 811 & Other & 0 & 0 \\
\hline 824 & 824 & Other & 0 & 0 \\
\hline 830 & B830 & Service & 13,878 & 0 \\
\hline 842 & 842 & & 0 & 0 \\
\hline 910 & 910 & Other & 0 & 0 \\
\hline 919 & 919 & & 0 & 0 \\
\hline 920 & 920 & & 0 & 0 \\
\hline 921 & 921 & & 0 & 0 \\
\hline 922 & B922 & Service & 0 & 0 \\
\hline 923 & B923 & Service & 0 & 0 \\
\hline 990 & B990 & Service & 296,790 & 23,426 \\
\hline 994 & B994 & Service & 372,923 & 0 \\
\hline 998 & 998 & Food sales & 0 & 0 \\
\hline 999 & 999 & Other & 0 & 0 \\
\hline 1000 & B1000 & Office & $3,300,000$ & 0 \\
\hline 1028 & B1028 & Service & 34,927 & 0 \\
\hline 1029 & B1029 & Service & $2,817,600$ & 0 \\
\hline 1031 & 1031 & Lodging & 0 & 0 \\
\hline 1032 & 1032 & Other & 68,300 & 1,896 \\
\hline 1036 & 1036 & Other & 0 & 0 \\
\hline 1070 & 1070 & Other & 0 & 0 \\
\hline 1081 & 1081 & & 129,040 & 0 \\
\hline 1082 & 1082 & & 316,775 & 0 \\
\hline 1085 & 1085 & & 0 & 0 \\
\hline 5001 & AKER & Lodging & 0 & 0 \\
\hline 5002 & APPTEC & Lodging & 0 & 0 \\
\hline 5003 & CRESCENT 1 & Lodging & $1,328,550$ & 0 \\
\hline 5004 & CRESCENT 3 & Lodging & $2,762,550$ & 0 \\
\hline 5005 & Crescent 5 & Lodging & 76,704 & 0 \\
\hline 5008 & QUARTERS A & Lodging & 23,064 & 0 \\
\hline 5009 & QUARTERS B & Lodging & 19,820 & 0 \\
\hline 5010 & QUARTERS C & Lodging & 58,360 & 0 \\
\hline 5011 & QUARTERS K & Lodging & 40,340 & 0 \\
\hline 5012 & QUARTERS L & Lodging & 30,407 & 0 \\
\hline 5013 & QUARTERS M & Lodging & 201,149 & 0 \\
\hline 5014 & QUARTERS M1 & Lodging & 48,593 & 0 \\
\hline 5015 & QUARTERS M2 & Lodging & 12,049 & 0 \\
\hline 5016 & QUARTERS M3 & Lodging & 6,850 & 0 \\
\hline 5017 & QUARTERS M4 & Lodging & 7,349 & 0 \\
\hline 5018 & QUARTERS M5 & Lodging & 4,808 & 0 \\
\hline
\end{tabular}




\begin{tabular}{|l|l|c|c|c|}
5019 & QUARTERS M6 & Lodging & 13,049 & 0 \\
\hline 5020 & QUARTERS M7 & Lodging & 118,600 & 0 \\
\hline 5021 & QUARTERS N & Lodging & 22,313 & 0 \\
\hline 5022 & QUARTERS O & Lodging & 74,180 & 0 \\
\hline 5028 & $\begin{array}{l}\text { STOCK EXCHANGE } \\
\text { BLDG. }\end{array}$ & Service & 0 & 0 \\
\hline 5029 & TASTYKAKE & Service & 0 & 0 \\
\hline 5030 & UNIQUE & Service & 0 & 0 \\
\hline
\end{tabular}

Table A5 Estimated Electric and Natural Gas use of the Navy Yard by Principal Building Activity

\begin{tabular}{|c|c|c|c|c|c|}
\cline { 2 - 6 } \multicolumn{1}{c|}{} & $\begin{array}{c}\text { annual Electric } \\
\text { use }(\mathrm{kWh})\end{array}$ & $\begin{array}{c}\text { annual Electric } \\
\text { use (MBtu) }\end{array}$ & $\begin{array}{c}\text { annual Natural } \\
\text { Gas use (CCF) }\end{array}$ & $\begin{array}{c}\text { annual Natural } \\
\text { Gas use (MBtu) }\end{array}$ & $\begin{array}{c}\text { Total Electric and } \\
\text { Natural Gas } \\
\text { (MBtu) }\end{array}$ \\
\hline food sales & $0.0 \mathrm{E}+00$ & 0.0 & $0.0 \mathrm{E}+00$ & 0.0 & 0.0 \\
\hline lodging & $4.8 \mathrm{E}+06$ & 16.5 & $0.0 \mathrm{E}+00$ & 0.0 & 16.5 \\
\hline office & $1.3 \mathrm{E}+07$ & 44.4 & $6.8 \mathrm{E}+04$ & 7.0 & 51.4 \\
\hline religious worship & $1.9 \mathrm{E}+04$ & 0.1 & $5.6 \mathrm{E}+03$ & 0.6 & 0.6 \\
\hline service & $6.9 \mathrm{E}+06$ & 23.5 & $1.2 \mathrm{E}+06$ & 118.5 & 142.0 \\
\hline $\begin{array}{c}\text { warehouse \& } \\
\text { storage }\end{array}$ & $3.5 \mathrm{E}+06$ & 11.8 & $2.2 \mathrm{E}+05$ & 22.6 & 34.4 \\
\hline $\begin{array}{c}\text { other (incl. non- } \\
\text { categorized) }\end{array}$ & $1.9 \mathrm{E}+07$ & 65.8 & $1.1 \mathrm{E}+05$ & 11.3 & 77.0 \\
\hline Total & $4.8 \mathrm{E}+07$ & 162.1 & $1.6 \mathrm{E}+06$ & 159.9 & 322.0 \\
\hline
\end{tabular}




\section{Appendix B: Building Type Definitions}

http://www.eia.gov/emeu/cbecs/building_types.html]

\begin{tabular}{|c|c|c|}
\hline Building Type & Definition & $\begin{array}{l}\text { Includes These Sub-Categories from } 2003 \text { CBECS } \\
\text { Questionnaire }\end{array}$ \\
\hline \multirow[t]{8}{*}{ Lodging } & \multirow{8}{*}{$\begin{array}{l}\text { Buildings used to offer multiple } \\
\text { accommodations for short-term or long- } \\
\text { term residents, including skilled nursing and } \\
\text { other residential care buildings. }\end{array}$} & motel or inn \\
\hline & & hotel \\
\hline & & dormitory, fraternity, or sorority \\
\hline & & retirement home \\
\hline & & nursing home, assisted living, or other residential care \\
\hline & & convent or monastery \\
\hline & & shelter, orphanage, or children's home \\
\hline & & halfway house \\
\hline \multirow[t]{12}{*}{ Office } & \multirow{12}{*}{$\begin{array}{l}\text { Buildings used for general office space, } \\
\text { professional office, or administrative offices. } \\
\text { Medical offices are included here if they do } \\
\text { not use any type of diagnostic medical } \\
\text { equipment (if they do, they are categorized } \\
\text { as an outpatient health care building). }\end{array}$} & administrative or professional office \\
\hline & & government office \\
\hline & & mixed-use office \\
\hline & & bank or other financial institution \\
\hline & & medical office (see previous column) \\
\hline & & sales office \\
\hline & & contractor's office (e.g. construction, plumbing, HVAC) \\
\hline & & non-profit or social services \\
\hline & & research and development \\
\hline & & city hall or city center \\
\hline & & religious office \\
\hline & & call center \\
\hline \multirow[t]{2}{*}{$\begin{array}{l}\text { Religious } \\
\text { Worship }\end{array}$} & \multirow{2}{*}{$\begin{array}{l}\text { Buildings in which people gather for } \\
\text { religious activities, (such as chapels, } \\
\text { churches, mosques, synagogues, and } \\
\text { temples). }\end{array}$} & No subcategories collected. \\
\hline & & \\
\hline
\end{tabular}




\begin{tabular}{|c|c|c|}
\hline \multirow[t]{12}{*}{ Service } & \multirow{12}{*}{$\begin{array}{l}\text { Buildings in which some type of service is } \\
\text { provided, other than food service or retail } \\
\text { sales of goods }\end{array}$} & vehicle service or vehicle repair shop \\
\hline & & vehicle storage/ maintenance (car barn) \\
\hline & & repair shop \\
\hline & & dry cleaner or laundromat \\
\hline & & post office or postal center \\
\hline & & car wash \\
\hline & & gas station \\
\hline & & photo processing shop \\
\hline & & beauty parlor or barber shop \\
\hline & & tanning salon \\
\hline & & copy center or printing shop \\
\hline & & kennel \\
\hline \multirow{3}{*}{$\begin{array}{l}\text { Warehouse and } \\
\text { Storage }\end{array}$} & \multirow{3}{*}{$\begin{array}{l}\text { Buildings used to store goods, manufactured } \\
\text { products, merchandise, raw materials, or } \\
\text { personal belongings (such as self-storage). }\end{array}$} & refrigerated warehouse \\
\hline & & non-refrigerated warehouse \\
\hline & & distribution or shipping center \\
\hline \multirow[t]{8}{*}{ Other } & \multirow{8}{*}{$\begin{array}{l}\text { Buildings that are industrial or agricultural } \\
\text { with some retail space; buildings having } \\
\text { several different commercial activities that, } \\
\text { together, comprise } 50 \text { percent or more of } \\
\text { the floorspace, but whose largest single } \\
\text { activity is agricultural, industrial/ } \\
\text { manufacturing, or residential; and all other } \\
\text { miscellaneous buildings that do not fit into } \\
\text { any other category. }\end{array}$} & airplane hangar \\
\hline & & crematorium \\
\hline & & laboratory \\
\hline & & telephone switching \\
\hline & & agricultural with some retail space \\
\hline & & manufacturing or industrial with some retail space \\
\hline & & data center or server farm \\
\hline & & \\
\hline
\end{tabular}

